\title{
No Gell-Mann and Low Eigenvalue in Quantum Electrodynamics.
}

\author{
R. ACHARYA
}

Physics Department, Arizona State University - Tempe, AZ 85287

P. Narayana Swamy

Physics Department, Southern Illinois University - Edwardsville, IL 62026

(Nuovo Cimento A, 103, 1131 (1990))

PACS 11.10.Gh - Renormalization.

PACS 11.10.Np - Gauge field theories.

PACS 12.20.Ds - Specific calculations and limits of quantum electrodynamics.

PACS 99.10 - Errata.

A phrase was left out in the abstract. The entire abstract should read as follows: It is argued that the Gell-Mann and Low eigenvalue equation, $\psi(x)=0$ in quantum electrodynamics does not possess a nontrivial solution, $x_{0} \neq 0$ and hence that quantum electrodynamics cannot be a finite field theory. This result reinforces the Baker-Johnson conjecture of 1979 , and is based on the ansatz that the longitudinal part of the vector vertex function dominates over the transverse piece in the infrared limit.

The paragraph after eq. (3.7) should be replaced by the following: We now make the following crucial and important ansatz: the unknown transverse piece of the vertex function which is unconstrained by the WT identity, (3.1), can be ignored in the infrared limit in a computation of the photon renormalization constant $Z_{3}^{-1}$. This can be demonstrated as follows.

The sentence following eq. (3.15) should read as follows: An inspection of eqs. (3.13) and (3.15) shows that the transverse parts of the vertex function which make explicit appearance in (3.11) make no contribution and the vertex function can thus be replaced by the longitudinal parts, provided that the left-hand sides of eqs. (3.13) and (3.15) vanish faster than $O(q)$ in the infrared limit. Henceforward we shall employ this ansatz. It may be recalled that the analogues of the left-hand sides of eqs. (3.13) and (3.15) for the longitudinal parts of the vertex funcion are in fact $O(q)$.

The last paragraph in sect. 4 should read as follows: In conclusion, the central result of this paper reinforces the 1979 conjecture of Baker and Johnson [23] that the Gell-Mann-Low function has no nontrivial zero in QED and hence [23] «that the finite theory does not seem to be a real possibility", provided that the longitudinal part of the vector vertex function dominates over the transverse piece in the infrared limit [24].

An additional footnote and reference should be inserted.

[24] This ansatz has been successfully employed in QED in other contexts. See, e.g., R. Delbourgo: Nuovo Cimento A, 49, 484 (1979). 